\section{BSG-Urteil: Bereitschaftsdienst geht alle an}

\author{
Der ärztliche Notdienst nachts, am Wochenende und an Feiertagen ist \\ Bestandteil vertragsärztlicher Versorgung. Wie aber diesen gewährleisten?
}

A uch spezialisierte Vertragsärzte kommen nicht um den Bereitschaftsdienst herum. Denn eine KV kann grundsätzlich alle zugelassenen Vertragsärzte zum Bereitschaftsdienst verpflichten, urteilte jetzt das Bundessozialgericht (BSG - B 6 KA 41/14 R). Mit Druck bis hin zum Zulassungsentzug können die KVen dafür sorgen, dass Vertragsärzte sich entsprechend fortbilden. Konkret wies das BSG einen Facharzt für Psychotherapeutische Medizin aus Niedersachsen ab, der bis 200713 Jahre lang vom Bereitschaftsdienst und den dafür notwendigen Fortbildungen befreit war. Er bekommt nun eine Übergangsfrist für seine Fortbildung von gut einem Jahr. Weigert er sich, darf die KV ihn nicht einteilen, kann aber disziplinarischen Druck ausüben. Bei einer dauerhaften Fortbildungsverweigerung „entfällt die Eignung für die Teilnahme an der vertragsärztlichen Versorgung insgesamt“, betonte der BSG-Vertragsarztsenat.

Nach einer Neuordnung des Bereitschaftsdienstes aus dem Jahre 2006 sollen in Niedersachsen alle approbierten Vertragsärzte am Bereitschaftsdienst teilnehmen. Ende 2007 teilte die KV dem betreffenden ärztlichen Psychotherapeuten mit, er müsse künftig am Notdienst teilnehmen und zunächst die erforderlichen Fortbildungen nachholen. Ab 2009 werde er zum Bereitschaftsdienst herangezogen. Dagegen wehrte

Homepanen werden Gruppierungen glied ungskagu.a. Aufnahm. Sie finden oder tungskalender.

Ärztlicher Notdienst

Warmbronn und Leonberg.

detfalldienst an Feiertarberg ist Gebersheim,

aus Leon Klinikverbund

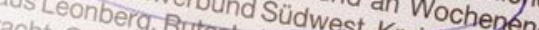

racht. Geöffnet ister heimer Straße 50 rasranken-

20 Uhr erfordelefonisc. 8 bis 8 Uhr des unterge-

e Nummer lautet

oranmeldung folgen-

Fraue,

unfrage

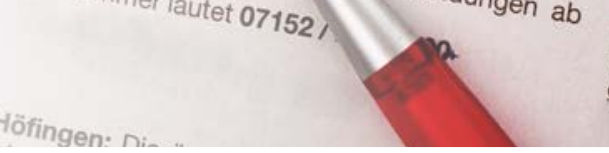

sich der Arzt mit dem Hinweis, er habe sich seit dem Ende seiner Ausbildung nur noch mit der Psychotherapie beschäftigt. Nicht alle KVen gehen so rigoros wie Niedersachsen vor, um die Pflicht zur Übernahme von Bereitschaftsdiensten durchzusetzen. Das hat eine Umfrage unter den KVen ergeben. (siehe auch www.aerztezeitung.de)

Martin Wortmann

\title{
Nicht nur Zuweiser bringen Patienten in die Praxis
}

\section{Stimmt der Praxisauftritt im Internet werden Suchmaschine und damit auch Patienten schnell fündig. Ärzte können dafür leicht in Vorleistung gehen.}

$\mathrm{O}$ b nach der Existenzgründung oder für die Expansion der Praxis - wer neue Patienten gewinnen will, kommt um Online-Aktivitäten beim Praxismarketing kaum noch herum. Darauf hat Praxisberaterin Nadja Alin Jung, $\mathrm{m} 2 \mathrm{c}$ medical concepts \& consulting, beim Seminar „Existenzgründung 2.0“ in Berlin hingewiesen. Immer mehr Patienten, die nach einem neuen Arzt suchen, konsultieren dafür das Internet. Dabei spielten Arztbewertungsportale ebenso eine Rolle wie eine Platzierung unter den ersten Einträgen bei der Suchmaschine Google für Leistungen der Praxis, sagte Jung bei der Veranstaltung, die von der Deutschen Apotheker- und Ärztebank gemeinsam mit der Steuerbe ratungsgesellschaft ETL Advision und Springer Medizin ausgerichtet wurde. „Bei Google auf Seite 1 - da spielt die $\mathrm{Mu}$ sik“, betonte die Praxisberaterin, „daher ist es wichtig, dass Ärzte lernen, mit diesen Medien zu arbeiten“.

Die zunehmende Bedeutung von Online-Medien für die Arztsuche zeigt sich auch in Umfragen: 2014 gaben $42 \%$ der Teilnehmer an einer Umfrage unter 1.000 Patienten im Auftrag des Bewertungsportals jameda an, Internet-Suchmaschinen für die Arztsuche zu nutzen, fast jeder dritte recherchierte zu diesem Zweck auf einem Arztbewertungsportal. Nur Freunde und Familie (56\%) und Hausärzte (41\%) waren für die Arztsuche wichtiger oder ebenso wichtig wie Suchmaschinen und Bewertungsportale.

Um bei Google weit vorne gelistet $\mathrm{zu}$ werden, sollten Ärzte auf ihrer Website mit Schlüsselworten arbeiten, die zu ihren Spezialleistungen, für die sie Patienten gewinnen wollen, passen. Auch substanzielle und verständlich geschriebene aktuelle Informationen $\mathrm{zu}$ diesen Leis- tungen helfen, bei Google nach vorne zu kommen. Interessierte Ärzte verfassen die Texte selber, es können aber auch Dienstleister konsultiert werden. Ob die Maßnahmen zum Erfolg führen, kann selbst getestet werden, etwa indem Suchbegriffe wie „Krebsvorsorge Frankfurt“ oder „Vasektomie Köln“ oder ähnliches selbst eingegeben werden - je nach Fachgruppe und speziell angebotenen Leistungen. Die Nutzung der eigenen Website lässt sich über Google Analytics recherchieren. Über Google Trends lässt sich erfahren, nach welchen Suchbegriffen wie oft gesucht wird.

Anzeigen bei Google seien dagegen weniger sinnvoll, habe sie festgestellt, sagte Marketing-Expertin Jung in Berlin. Dagegen könne es sehr wohl helfen, zufriedene Patienten anzuregen, die Praxis im Netz auf einschlägigen Portalen zu bewerten. Wichtig sei es auch, regelmäßig zu screenen, wie die Praxis bewertet werde, um gegebenenfalls Missstände abzustellen, auf die Patienten im Internet hinweisen.

Hauke Gerlof

Das nächste Seminar Existenzgründung 2.0 für Ärzte findet am 24. Oktober 2015 in München statt: www.apoBank.de/existenzgruendung-2-0 